\title{
THE BEHAVIOR AT INFINITY OF CERTAIN CONVOLUTION TRANSFORMS $\left({ }^{(1)}\right.$
}

BY

\author{
I. I. HIRSCHMAN, JR.
}

1. Introduction. Let $b,\left\{a_{k}\right\}_{1}^{\infty}$ be real constants subject to the sole restriction that

$$
\sum_{k=1}^{\infty}{\overrightarrow{a_{k}}}^{2}<\infty
$$

and let

$$
E(s)=e^{b s} \prod_{1}^{\infty}\left(1-\frac{s}{a_{k}}\right) e^{s / a_{k}}
$$

We define

$$
G(t)=\frac{1}{2 \pi i} \int_{-i \infty}^{i \infty} \frac{e^{s t}}{E(s)} d s \quad(-\infty<t<\infty) .
$$

It may be verified that

$$
\int_{-\infty}^{\infty} G(\dot{t}) e^{-s t} d s=\frac{1}{E(s)}
$$

the bilateral Laplace transform converging absolutely in the strip $\left(\alpha_{1}<\sigma<\alpha_{2}\right)$ where

$$
\alpha_{2}=\operatorname{Min}_{a_{k}>0} a_{k}, \quad \alpha_{1}=\operatorname{Max}_{a_{k}<0} a_{k},
$$

and $s=\sigma+i \tau$. The function $G(t)$ and the associated convolution transform

$$
f(x)=\int_{-\infty}^{\infty} G(x-t) d \alpha(t)
$$

have been studied in [2], [3], and [4](2).

A kernel $G(t)$ belongs to class II if the constants $a_{k}$ are positive and if $\sum_{1}^{\infty} 1 / a_{k}=\infty$. If $G(t) \in$ class II and if the convolution transform (6) converges for any one value of $x$ then it converges for all larger values of $x$. Consequently there exists a number $\gamma_{c}$, the abscissa of convergence, such that the

Presented to the Society, September 10, 1948; received by the editors November 4, 1948.

(1) This research was supported in part by the Office of Naval Research under Contract NR 043-052.

(2) Numbers in brackets refer to the bibliography at the end of the paper. 
transform (6) converges for $x>\gamma_{c}$ and diverges for $x<\gamma_{c}$.

Our principal result is that if $f(x)$ is the generating function of a convolution transform with class II kernel $G(t)$ and determining function $\alpha(t)$, and if

$$
f(x)=O[G(x-\rho)] \quad(x \rightarrow+\infty)
$$

then $\alpha(t)$ is necessarily constant for $t>\rho$, so that

$$
f(x)=\int_{-\infty}^{\rho+\epsilon} G(x-t) d \alpha(t)
$$

for every $\epsilon>0$. If (7) holds for arbitrarily large negative $\rho$ then $f(x) \equiv 0$.

As an example we set

$$
E(s)=[\Gamma(1-s)]^{-1}, \quad G(t)=e^{-e t} e^{t} .
$$

If

$$
\begin{aligned}
f(x) & =\int_{-\infty}^{\infty} e^{-e^{x-t} e^{x-t} \phi(t) d t} & \left(x>\gamma_{c}\right), \\
f(x) & =O\left[e^{-e^{x-\rho}} e^{x-\rho}\right] & (x \rightarrow+\infty),
\end{aligned}
$$

then $\phi(t)=0$ for $(\rho<t<\infty)$. After an exponential change of variable this becomes a theorem concerning the Laplace transform. If

$$
\begin{array}{lrl}
F(x)=\int_{0}^{\infty} e^{-x t} \Phi(t) d t & \left(x>g_{c}\right), \\
F(x)=O\left(e^{-r x}\right) & (x \rightarrow+\infty),
\end{array}
$$

then $\Phi(t)=0$ for $(0<t<r)$. A proof of this special case has been given previously in [1].

A function $G(t)$ is said to belong to class Ia if there are both positive and negative $a_{k}$ 's and if $\sum_{1}^{\infty} 1 /\left|a_{k}\right|=\infty$. If $G(t) \in$ class Ia and if the convolution transform (6) converges for any one value of $x$, it converges for all $x$. With each kernel $G(t)$ class Ia we may associate a class II kernel $\bar{G}(t)$ defined by the equations

$$
\begin{array}{ll}
\bar{E}(s)=e^{b s} \prod_{1}^{\infty}\left(1-\frac{s}{\left|a_{k}\right|}\right) e^{s|| a_{k} \mid,} \\
\bar{G}(t)=\frac{1}{2 \pi i} \int_{-i \infty}^{i \infty} \frac{e^{s t}}{\bar{E}(s)} d s \quad(-\infty<t<\infty) .
\end{array}
$$

We shall also prove that if $f(x)$ is a generating function with kernel $G(t) \in$ class Ia and if

$$
f(x)=O[\bar{G}(x-\rho)] \quad(x \rightarrow+\infty)
$$


for arbitrarily large negative $\rho$, or

$$
f(x)=O[\bar{G}(\rho-x)]
$$$$
(x \rightarrow-\infty)
$$

for arbitrarily large positive $\rho$, then $f(x) \equiv 0$.

As an example of this result we may take

$$
E(s)=\cos \pi s, \quad G(t)=\operatorname{sech} \frac{t}{2} .
$$

After an exponential change of variables we obtain a theorem concerning the Stieltjes transform. If

$$
F(x)=\int_{0+}^{\infty} \frac{\Phi(t)}{x+t} d t \quad(0<x<\infty)
$$

and if

$$
F(x)=O\left(e^{-r x x^{1 / 2}}\right) \quad(x \rightarrow+\infty)
$$

or if

$$
F(x)=O\left(e^{-r x-1 / 2}\right) \quad(x \rightarrow 0+)
$$

for arbitrarily large $r$, then $F(x) \equiv 0$.

2. A special case. In this section we shall prove our first theorem under very special assumptions. The most general case can, however, be reduced to this one by elementary transformations.

\section{THEOREM 2. If}

1. $G(t) \in$ class II,

2. $\phi(t) \in L(\rho \leqq t \leqq \infty)$,

3. $h(x)=\int_{\rho}^{\infty} G(x-t) e^{c t} \phi(t) d t\left(c<\alpha_{2}\right)$, where $\alpha_{2}$ is defined as in (5) $\$ 1$, then

4. $h(x)=O[G(x-\rho)]$

$$
\phi(t) \equiv 0 \quad(\rho<t<\infty) .
$$

We define

$$
\phi^{*}(s)=\int_{\rho}^{\infty} e^{c t} \phi(t) e^{-s t} d t
$$

This bilateral Laplace transform converges absolutely for $(c \leqq \sigma<\infty)$. We know that

$$
\frac{1}{E(s)}=\int_{-\infty}^{\infty} G(t) e^{-s t} d t
$$

the integral converging absolutely for $\left(-\infty<\sigma / \alpha_{2}\right)$. 
Since $G(t) e^{-c t}$ is positive and bell-shaped, see $[3 ; \S 10]$, we have for $x$ sufficiently large and negative

$$
\begin{aligned}
|h(x)| & \leqq e^{c x}\left[\operatorname{Max}_{\rho \leqq \infty} G(x-t) e^{-c(x-t)}\right] \int_{\rho}^{\infty}|\phi(t)| d t \\
& =O[G(x-\rho)] \quad(x \rightarrow-\infty) .
\end{aligned}
$$

From this estimation and from assumption 4 it follows that

$$
|h(x)| \leqq O(1) G(x-\rho) \quad(-\infty<x<\infty) .
$$

This implies that the bilateral Laplace transform

$$
h^{*}(s)=\int_{-\infty}^{\infty} h(x) e^{-s x} d x
$$

converges absolutely for $\left(-\infty<\sigma<\alpha_{2}\right)$.

Since the integrals (1) and (2) have a common strip of absolute convergence $\left(c<\sigma<\alpha_{2}\right)$, the convolution theorem for the bilateral Laplace transform tells us that in this strip

or

$$
h^{*}(s)=\frac{1}{E(s)} \phi^{*}(s),
$$

$$
\phi^{*}(s)=h^{*}(s) E(s) .
$$

Since $E(s)$ is an entire function, equation (5) provides a continuation of $\phi^{*}(s)$ into the half-plane $\sigma \leqq c$, so that $\phi^{*}(s)$ is also an entire function.

By a change of variable in equation (1) we obtain

$$
e^{\rho(s-c)} \phi^{*}(s)=\int_{0}^{\infty} e^{c t} \phi(t+\rho) e^{-s t} d t .
$$

It is clear that $e^{\rho s} \phi^{*}(s)$ is bounded in the half-plane $\sigma \geqq c$. In particular $e^{\rho s} \phi^{*}(s)$ is bounded on the line $(\sigma=c,-\infty<\tau<\infty)$.

Using inequality (3) we have

$$
\left|h^{*}(s)\right|=O(1)\left[E(\sigma) e^{\rho \sigma}\right]^{-1} \quad\left(-\infty<\sigma<\alpha_{2}\right)
$$

from which we obtain

$$
\left|e^{\rho s} \phi^{*}(s)\right| \leqq O(1)\left|\frac{E(s)}{E(\sigma)}\right| \quad\left(-\infty<\sigma<\alpha_{2}\right) .
$$

It follows that $e^{\rho s} \phi^{*}(s)$ is bounded on the half-line $(-\infty<\sigma \leqq c, \tau=0)$ and that in the half-plane $\left(-\infty<c \leqq \sigma, \phi^{*}(s)\right)$ is at most of order two minimal type.

We now know that $e^{\rho s} \phi^{*}(s)$ is bounded on the lines $(\sigma=c,-\infty<\tau<\infty)$ and $(-\infty<\sigma \leqq c, \tau=0)$ and is at most of order two minimal type for $\sigma \leqq c$. 
Applying a familiar form of the Phragmén-Lindelöf principle to each of the two quadrants of this configuration we find that $e^{\rho s} \phi^{*}(s)$ is bounded for $\sigma \leqq c$ and thus in the entire plane. By Liouville's theorem $e^{\rho s} \phi^{*}(s)$, being entire and bounded, is a constant. Since

$$
\begin{array}{rlrl}
e^{i \rho \tau} \phi^{*}(c+i \tau) & =\int_{0}^{\infty} \phi(t+\rho) e^{-i t \tau} d t & \\
& =o(1) & & (\tau \rightarrow \pm \infty)
\end{array}
$$

it follows that $\phi^{*}(s) \equiv 0$. This implies that $\phi(t)=0$ almost everywhere $(\rho \leqq t$ $<\infty)$. q.e.d.

3. Two lemmas. We consider here two lemmas necessary to reduce the general case to the special case which we have just considered. Let

$$
f(x)=\int_{-\infty}^{\infty} G(x-t) d \alpha(t)
$$

have abscissa of convergence $\gamma_{c}$. We set

$$
\begin{aligned}
& A_{1}(t)=a_{1} e^{a_{1} t} \int_{t}^{\infty} e^{-a_{1} u} d \alpha(u), \\
& A_{m}(t)=a_{m} e^{a_{m} t} \int_{t}^{\infty} e^{-a_{m} u} A_{m-1}(u) d u \quad(m=2, \cdots, n)
\end{aligned}
$$

where $n$ is chosen so large that $s-\alpha_{2}$ is not a zero of

$$
\prod_{n+1}^{\infty}\left(1-\frac{s}{a_{k}}\right) e^{s / a_{k}}
$$

We further define the auxiliary kernel

$$
H(t)=\frac{1}{2 \pi i} \int_{-i \infty}^{i \infty} \frac{\exp \left(s\left(t-\sum_{1}^{n} a_{k}^{-1}\right)\right)}{e^{b s} \prod_{n+1}^{\infty}\left(1-\left(s / a_{k}\right)\right) e^{s / a_{k}}} d s \quad(-\infty<t<\infty) .
$$

LEMMA 3a. If $f(x)$ is given by (1) and if $A_{n}(t)$ and $H(t)$ are defined as in (2) and (3) then

A.

$$
\begin{aligned}
f(x) & =\int_{-\infty}^{\infty} H(x-t) A_{n}(t) d t & \left(x>\gamma_{c}\right), \\
A_{n}(t) & =o\left(e^{\alpha_{2} t}\right) & (t \rightarrow+\infty) .
\end{aligned}
$$

B.

The convergence of the integral (1) insures that $A_{1}(t)$ is defined, see [3; \$15]. Writing

$$
f(x)=\int_{-\infty}^{\infty} G(x-t) d \alpha(t)=\int_{-\infty}^{\infty} G(x-t) e^{a_{1} t} e^{-a_{1} t} d \alpha(t)
$$


and integrating by parts we have

$$
\begin{aligned}
\int_{-\infty}^{\infty} G(x-t) d \alpha(t)= & {\left[-G(x-t) e^{a_{1} t} \int_{t}^{\infty} e^{-a_{1} u} d \alpha(u)\right]_{-\infty}^{\infty} } \\
& +\int_{-\infty}^{\infty} A_{1}(t)\left[\frac{1}{a_{1}} e^{-a_{1} t} \frac{d}{d t} e^{a_{1} t} G(x-t)\right] d t .
\end{aligned}
$$

It is easily verified that for $x>\gamma_{c}$ the integrated term vanishes; see [ $3 ; \S \S 16$, 20]. Thus

$$
f(x)=\int_{-\infty}^{\infty}\left\{\left[1+\frac{D}{a_{1}}\right] G(x-t)\right\} A_{1}(t) d t \quad\left(x>\gamma_{c}\right) .
$$

The convergence of the integral (4) insures that $A_{2}(t)$ is defined, and so forth. After $n$ such steps we obtain

$$
\begin{aligned}
f(x) & =\int_{-\infty}^{\infty}\left\{\prod_{1}^{n}\left(1+\frac{D}{a_{k}}\right) G(x-t)\right\} A_{n}(t) d t \\
& =\int_{-\infty}^{\infty} H(x-t) A_{n}(t) d t,
\end{aligned}
$$

which is conclusion $\mathrm{A}$.

We may establish conclusion B by induction. We know that $I(t)$ $=\int_{i}^{\infty} e^{-\alpha_{2} u} d \alpha(u)$ converges. If $\alpha_{2}=a_{1}$, conclusion $\mathrm{B}$ is obvious for $n=1$. If $\alpha_{2}<a_{1}$, then

$$
\begin{array}{rlrl}
A_{1}(t) & =a_{1} e^{a_{1} t}\left\{\left[-e^{-a_{1} u+\alpha_{2} u} I(u)\right]_{t}^{\infty}+\left(\alpha_{2}-a_{1}\right) \int_{t}^{\infty} e^{-a_{1} u+\alpha_{2} u} I(u) d u\right\}, \\
& =o\left(e^{\alpha_{2} t}\right) & & (t \rightarrow+\infty) .
\end{array}
$$

Suppose now that our result is proved for $m-1$. We will establish it for $m$. If $a_{m}=\alpha_{2}$ it is obvious. If $a_{m}>\alpha_{2}$, then using our induction assumption we see that

$$
\begin{aligned}
A_{m} & =o\left(e^{a_{m} u} \int_{t}^{\infty} e^{-a_{m} u} e^{\alpha_{2} u} d u\right) \\
& =o\left(e^{\alpha_{2} t}\right)
\end{aligned}
$$$$
(t \rightarrow+\infty)
$$

Conclusion B now follows.

LEMMa $3 \mathrm{~b}$. If

1. $G(t) \in$ class II, then

2. $f(x)=\int_{-\infty}^{\infty} G(x-t) d \alpha(t)$ has abscissa of convergence $\gamma_{c}$, 


$$
\int_{-\infty}^{\rho} G(x-t) d \alpha(t)=O[G(x-\rho)] \quad(x \rightarrow+\infty) .
$$

If $\delta$ is any number greater than $\gamma_{c}$ then we know, see $[3 ; \$ \$ 16,20]$, that there is a positive constant $A$ such that

$$
|\alpha(t)| \leqq A e^{x(\delta-t)} \quad(-\infty<t \leqq \rho),
$$

where $\chi(t)=-\log G(t)$. Integrating by parts we find that for $x>\delta$

$$
\int_{-\infty}^{\rho} G(x-t) d \alpha(t)=\int_{-\infty}^{\rho} \chi^{\prime}(x-t) e^{-\chi(x-t)} \alpha(t) d t+O[G(x-\rho)] .
$$

For $x$ sufficiently large $\chi^{\prime}(x-t)$ is of constant (positive) sign in $(-\infty<t<\rho)$ therefore

$$
\left|\int_{-\infty}^{\rho} \chi^{\prime}(x-t) e^{-\chi(x-t)} \alpha(t) d t\right| \leqq A \int_{-\infty}^{\rho} \chi^{\prime}(x-t) e^{-\chi(x-t)+\chi(\delta-t)} d t .
$$

Integrating by parts again,

$$
\begin{aligned}
\int_{-\infty}^{\rho} x^{\prime}(x-t) e^{-\chi(x-t)+x(\delta-t)} & \\
= & {\left[e^{-\chi(x-t)+x(\delta-t)}\right]_{-\infty}^{\rho}+\int_{-\infty}^{\rho} \chi^{\prime}(\delta-t) e^{-\chi(x-t)+x(\delta-t)} d t . }
\end{aligned}
$$

Now

$$
\left[e^{-x(x-t)+x(\delta-t)}\right]_{-\infty}^{\rho}=G(x-\rho) / G(\delta-\rho) .
$$

Further if $\left(\theta>\delta>\gamma_{c}\right)$, then

$$
\begin{aligned}
& \int_{-\infty}^{\rho} \chi^{\prime}(\delta-t) e^{-\chi(x-t)+\chi(\delta-t)} d t \\
& \quad=\int_{-\infty}^{\rho} \chi^{\prime}(\delta-t) e^{x(\delta-t)-x(\theta-t)} e^{x(\theta-t)-x(x-t)} d t \\
& \leqq \sup _{-\infty<t \leqq \rho}[G(x-t) / G(\theta-t)] \int_{-\infty}^{\rho}\left|\chi^{\prime}(\delta-t)\right| e^{x(\delta-t)-x(\theta-t)} d t .
\end{aligned}
$$

It may be verified that for $x$ sufficiently large,

$$
\sup _{-\infty<t \leqq \rho}[G(x-t) / G(\theta-t)]=G(x-\rho) / G(\theta-\rho),
$$

see $[3 ; \S 20]$. Thus 


$$
\int_{-\infty}^{\rho}\left|\chi^{\prime}(\delta-t)\right| e^{\chi(\delta-t)-\chi(x-t)} d t=O[G(x-\rho)] \quad(x \rightarrow+\infty) .
$$

Combining our results we have as desired,

$$
\int_{-\infty}^{\rho} G(x-t) d \alpha(t)=O[G(x-\rho)] \quad(x \rightarrow+\infty) .
$$

\section{The general case, $G(t) \in$ class II.}

\section{THEOREM 4. If}

1. $G(t) \in$ class II,

2. $f(x)=\int_{-\infty}^{\infty} G(x-t) d \alpha(t)$ has abscissa of convergence $\boldsymbol{\gamma}_{c}$,

3. $f(x)=O[G(x-\rho)](x \rightarrow+\infty)$, then $\alpha(t)$ is constant for $\rho<t<\infty$.

By conclusion A of Lemma 3a we have

$$
f(x)=\int_{-\infty}^{\infty} H(x-t) A_{n}(t) d t
$$

where $H$ and $A_{n}$ are defined in $\$ 3$. It is known $[3 ; \S \S 20,21]$ that if $\epsilon>0$

$$
\lim _{x \rightarrow+\infty} G(x-\rho) / H(x-\rho-\epsilon)=0 .
$$

Thus

$$
f(x)=O[H(x-\rho-\epsilon)] \quad(x \rightarrow+\infty) .
$$

By Lemma $3 \mathrm{~b}$ and equation (1)

$$
\int_{\rho+\epsilon}^{\infty} H(x-t) A_{n}(t) d t=O[H(x-\rho-\epsilon)] \quad(x \rightarrow+\infty) .
$$

If we choose $c\left(\alpha_{2}<c<\operatorname{Min}_{k>n} a_{k}\right)$, then by conclusion B of Lemma 3a

$$
\int_{\rho+\epsilon}^{\infty} H(x-t) A_{n}(t) d t=\int_{\rho+\epsilon}^{\infty} H(x-t) e^{c t}\left[e^{-c t} A_{n}(t)\right] d t,
$$

where $e^{-c t} A_{n}(t) \in L(\rho+\epsilon, \infty)$. We may apply Theorem 2 to conclude that $A_{n}(t)=0(\rho+\epsilon<t<\infty)$. Since $\epsilon$ is arbitrary $A_{n}(t)=0(\rho<t<\infty)$. Using the definition of $A_{n}(t)$ it is easily seen that $\alpha(t)$ is constant for $(\rho<t<\infty)$. q.e.d.

5. $G(t) \in$ class Ia. In order to illustrate the essential point of the argument which follows let us consider a very special case. Let $G(t) \in$ class Ia and let

$$
f(x)=\int_{-\infty}^{\infty} G(x-t) \phi(t) d t
$$

where $\phi(t) \in L^{2}(-\infty, \infty)$. We shall show that if 


$$
f(x)=O(\bar{G}(x-\rho))
$$$$
(x \rightarrow+\infty)
$$

for arbitrarily large negative $\rho$ then $f(x) \equiv 0$. Let $\mathfrak{F}$ denote the Fourier transformation

$$
\mathfrak{F}_{t} \psi=\frac{1}{(2 \pi)^{1 / 2}} \underset{T \rightarrow \infty}{\lim } \int_{-T}^{T} \psi(u) e^{i t u} d u
$$

and let $\mathfrak{F}_{t}^{-1}$ denote its inverse

$$
\mathfrak{F}_{t}^{-1} \psi=\frac{1}{(2 \pi)^{1 / 2}} \underset{T \rightarrow \infty}{\lim } \underset{T \rightarrow T}{(2)} \int_{-T}^{T} \psi(u) e^{-i t u} d u
$$

We assert that

$$
f(x)=\int_{-\infty}^{\infty} \bar{G}(x-t)\left[\mathfrak{F}_{t}^{-1}\left(\frac{E(-i u)}{\bar{E}(-i u)} \mathfrak{F}_{u} \phi(t)\right)\right] d t .
$$

From equation (1) we see that the Fourier transform of $f(x)$ satisfies the equation $\mathfrak{F}_{u} f=E(-i u) \mathfrak{F}_{u} \phi$. Computing the Fourier transform of the right-hand side of equation (3) we obtain

$$
\bar{E}(-i u) \mathfrak{F}_{u} \mathfrak{F}_{t}^{-1}\left[\frac{E(-i u)}{\bar{E}(-i u)} \mathfrak{F}_{u} \phi\right]=E(-i u) \mathfrak{F}_{u} \phi=\mathfrak{F}_{u} f
$$

The validity of equation (3) follows. By equations (2) and (3) and Theorem 4 $f(x) \equiv 0$, which is what we wished to show. The transformation

$$
\phi(t) \rightarrow \mathfrak{F}_{t}^{-1}\left[\frac{E(-i u)}{\bar{E}(-i u)} \mathfrak{F}_{u} \phi\right]
$$

is a Watson transformation. Our procedure in the general case is an adaptation of this argument.

LEMMA 5a. If

1. $G(t) \in$ class Ia,

2. $\left|\beta_{1}\right|,\left|\beta_{2}\right|<\operatorname{Min}\left[-\alpha_{1}, \alpha_{2}\right]$,

3. $\phi(t)=O\left(e^{\beta_{2} t}\right)(t \rightarrow+\infty)=O\left(e^{\beta_{1} t}\right)(t \rightarrow-\infty)$,

4. $f(x)=\int_{-\infty}^{\infty} G(x-t) \phi(t) d t$,

5. $f(x)=O[\bar{G}(x-\rho)]$ as $x \rightarrow+\infty$ for arbitrarily large negative $\rho$, then $f(x) \equiv 0$.

Choose any constant $c, c \geqq 2 \alpha_{\mathbf{2}}$. We define

$$
\begin{gathered}
F(s)=\exp \left\{\left[b+\left(\left|a_{1}\right|+c\right)^{-1}+\left(\left|a_{2}\right|+c\right)^{-1}\right] s\right\} \\
\cdot \prod_{n=3}^{\infty}\left(1-\frac{s}{\left|a_{n}\right|+c}\right) e^{s /\left(\left|a_{n}\right|+c\right),}
\end{gathered}
$$




$$
\begin{aligned}
K(t) & =\frac{1}{2 \pi i} \int_{-i \infty}^{i \infty} \frac{e^{s t}}{F(s)} d s \\
J(t) & =\frac{1}{2 \pi i} \int_{-i \infty}^{i \infty} \frac{F(s)}{E(s)} e^{s t} d s
\end{aligned}
$$$$
(-\infty<t<\infty) \text {, }
$$$$
(-\infty<t<\infty)
$$

The function $K(t)$ is a class II kernel. Let $\eta>0$ be so small that $\alpha_{1}+\eta<\beta_{1}$ and $\alpha_{2}-\eta>\beta_{2}$. By $[3 ; \S 9]$

$$
\begin{aligned}
K(t) & =O\left[e^{\left(\alpha_{2}-\eta\right) t}\right] & & (t \rightarrow-\infty) \\
& =O\left[e^{-k t}\right] & & (t \rightarrow+\infty)
\end{aligned}
$$

for every positive $K$. Further,

$$
\int_{-\infty}^{\infty} e^{-s t} K(t) d t=\frac{1}{F(s)}
$$

the bilateral Laplace transform converging absolutely for $\left(-\infty<\sigma<\alpha_{2}\right)$.

For $c$ chosen as above and for any $\sigma\left(\alpha_{1}<\sigma<\alpha_{2}\right)$, it may be verified that

$$
\left|1-\frac{\sigma+i \tau}{\left|a_{n}\right|+c}\right|\left|1-\frac{\sigma+i \tau}{a_{n}}\right|^{-1}
$$

decreases as $|\tau|$ increases. Let $\alpha_{1}<\sigma_{1} \leqq \sigma \leqq \sigma_{2}<\alpha_{2}$ be any proper subinterval of $\left(\alpha_{1}<\sigma<\alpha_{2}\right)$, and let

$$
M=\operatorname{Max}_{\sigma_{1} \leqq \sigma_{\sigma_{2}}}|F(\sigma) / E(\sigma)|
$$

We have

$$
\left|\frac{F(s)}{E(s)}\right| \leqq\left|\left(1-\frac{s}{a_{1}}\right)\left(1-\frac{s}{a_{2}}\right)\right|^{-1} \cdot M \quad\left(\sigma_{1} \leqq \sigma \leqq \sigma_{2}\right) .
$$

It follows that

$$
\left|\frac{F(s)}{E(s)}\right|=O\left(\frac{1}{\tau^{2}}\right)
$$

$$
(\tau \rightarrow \pm \infty)
$$

uniformly in $\sigma$ for $\sigma$ in any proper subinterval of $\left(\alpha_{1}<\sigma<\alpha_{2}\right)$. Because of this order relation we may deform the line of integration of the integral (3) to $\sigma=\alpha_{2}-\eta$ or to $\sigma=\alpha_{1}+\eta$. We obtain

$$
\begin{aligned}
J(t) & =\frac{1}{2 \pi i} \int_{\alpha_{2}-\eta-i \infty}^{\alpha_{2}-\eta+i \infty} \frac{F(s)}{E(s)} e^{s t} d s \\
& =\frac{1}{2 \pi i} \int_{\alpha_{1}+\eta-i \infty}^{\alpha_{1}+\eta+i \infty} \frac{F(s)}{E(s)} e^{s t} d s,
\end{aligned}
$$


and these together with equation (6) imply that

$$
\begin{array}{rlrl}
J(t) & =O\left(e^{\left(\alpha_{2}-\eta\right) t}\right) & & (t \rightarrow-\infty) \\
& =O\left(e^{\left(\alpha_{1}+\eta\right) t}\right) & (t \rightarrow+\infty) .
\end{array}
$$

Further, a simple application of a theorem of Hamburger, see [6; pp. 265266] shows that

$$
\int_{-\infty}^{\infty} J(t) e^{-s t} d t=\frac{F(s)}{E(s)}
$$

the integral converging absolutely for $\left(\alpha_{1}<\sigma<\alpha_{2}\right)$.

From equations (5) and (8) and the convolution theorem for the bilateral Laplace transform we have

$$
\int_{-\infty}^{\infty} K(x-t) J(t) d t=G(x) \quad(-\infty<x<\infty) .
$$

Consequently we may write

$$
f(x)=\int_{-\infty}^{\infty} G(x-t) \phi(t) d t=\int_{-\infty}^{\infty} \phi(t) d t \int_{-\infty}^{\infty} K(x-u) J(u-t) d u
$$

Because of the order conditions (4) and (7) and assumption 3 we may invert the order of integration to obtain

$$
f(x)=\int_{-\infty}^{\infty} K(x-u) \Phi(u) d u,
$$

where

$$
\Phi(u)=\int_{-\infty}^{\infty} J(u-t) \phi(t) d t
$$

Consider the class II kernel $L(t)$,

$$
L(t)=\frac{1}{2 \pi i} \int_{-i \infty}^{i \infty} \frac{e^{s t}}{e^{b s} \prod_{1}^{\infty}\left(1-s /\left(\left|a_{k}\right|+c\right)\right) e^{s /\left(\left|a_{k}\right|+c\right)}} d s \quad(-\infty<t<\infty) .
$$

It may be shown, see $[3 ; \S 20]$, that

$$
\bar{G}(t)=\left[\prod_{1}^{\infty}\left(1+\frac{c}{\left|a_{k}\right|}\right) e^{-c|| a_{k} \mid}\right]^{-1} e^{-c t L}\left(t-\sum_{1}^{\infty} \frac{c}{\left|a_{k}\right|\left(\left|a_{k}\right|+c\right)}\right) .
$$

Assumption 5 thus implies that $f(x)=O[L(x-\rho)](x \rightarrow+\infty)$ for arbitrarily large negative $\rho$. It follows from $[3 ; \S \S 20,21]$ that if $\epsilon>0$ then

$$
\lim _{t \rightarrow \infty} K(t) / L(t+\epsilon)=\infty \text {. }
$$


Hence

$$
f(x)=O[K(x-\rho)]
$$$$
(x \rightarrow+\infty)
$$

for arbitrarily large negative $\rho$. Equations (11) and (12) and Theorem 4 imply that

$$
f(x) \equiv 0,
$$

which is what we wished to prove.

THEOREM 5b. If

1. $G(t) \in$ class Ia,

2. $f(x)=\int_{-\infty}^{\infty} G(x-t) d \alpha(t)$,

3. $f(x)=O[\bar{G}(x-\rho)](x \rightarrow+\infty)$ for arbitrarily large negative $\rho$ where $\bar{G}$ is defined as in $\$ 1$,

then

$$
f(x) \equiv 0 \text {. }
$$

Choose $n$ so large that $s-\alpha_{1}$ and $s-\alpha_{2}$ are no longer zeros of

$$
\prod_{n+1}^{\infty}\left(1-\frac{s}{a_{k}}\right) e^{s / a_{k}} \text {, }
$$

and so large that if $\alpha_{3}=\operatorname{Min}\left|a_{k}\right|, k=n+1, n+2, \cdots$, then $\alpha_{3}>\operatorname{Max}$ $\left[-\alpha_{1}, \alpha_{2}\right]$. Let

$$
\begin{aligned}
& H(t)=\frac{1}{2 \pi i} \int_{-i \infty}^{i \infty} \frac{\exp \left(s\left[t-\sum_{1}^{n} a_{k}^{-1}\right]\right)}{e^{b s} \prod_{n+1}^{\infty}\left(1-s / a_{k}\right) e^{s / a_{k}}} d s, \\
& \bar{H}(t)=\frac{1}{2 \pi i} \int_{-i \infty}^{i \infty} \frac{\exp \left(s\left[t-\sum_{1}^{n} a_{k}^{-1}\right]\right)}{e^{b s} \prod_{n+1}^{\infty}\left(1-s /\left|a_{k}\right|\right) e^{s /\left|a_{k}\right|}} d s .
\end{aligned}
$$

Further we set

$$
\begin{aligned}
A_{1}(t) & =a_{1} e^{a_{1} t} \int_{t}^{\infty} e^{-a_{1} u} d \alpha(u) & & \text { (if } \left.a_{1}>0\right) \\
& =a_{1} e^{a_{1} t} \int_{-\infty}^{t} e^{-a_{1} u} d \alpha(u) & & \text { (if } \left.a_{1}<0\right), \\
A_{m}(t) & =a_{m} e^{a_{m} t} \int_{t}^{\infty} e^{-a_{m} u} A_{m-1}(u) d u & & \text { (if } \left.a_{m}>0\right) \\
& =a_{m} e^{a_{m} t} \int_{-\infty}^{t} e^{-a_{m} u} A_{m-1}(u) d u & & \text { (if } \left.a_{m}<0\right),
\end{aligned}
$$

for $m=2, \cdots, n$. Just as in Lemma 3 we may show that 


$$
f(x)=\int_{-\infty}^{\infty} H(x-t) A_{n}(t) d t
$$

and that

$$
\begin{aligned}
A_{n}(t) & =o\left(e^{\alpha_{2} t}\right) & & (t \rightarrow+\infty) \\
& =o\left(e^{\alpha_{1} t}\right) & & (t \rightarrow-\infty) .
\end{aligned}
$$

By our choice of $n$

$$
H(t)=O\left(e^{-\alpha_{3}|t|}\right) \quad(t \rightarrow+\infty)
$$

where $\alpha_{3}>\operatorname{Max}\left[-\alpha_{1}, \alpha_{2}\right]$. It may be shown, see $[3 ; \S \S 20,21]$ that if $\epsilon>0$ then

$$
\lim _{x \rightarrow+\infty} \bar{G}(t) / \bar{H}(t-\epsilon)=0 .
$$

Hence assumption 3 implies that

$$
f(x)=O(\bar{H}(x-\rho)) \quad(x \rightarrow+\infty)
$$

for arbitrarily large negative $\rho$. We may now apply Lemma 5 a to conclude that $f(x) \equiv 0$.

Similarly we may prove

THEOREM 5c. If

1. $G(t) \in$ class Ia,

2. $f(x)=\int_{-\infty}^{\infty} G(x-t) d \alpha(t)$,

3. $f(x)=\bar{G}(\rho-x)(x \rightarrow-\infty)$ for arbitrarily large positive $\rho$, then

$$
f(x) \equiv 0 \text {. }
$$

6. Class III kernels. A kernel $G(t)$ is said to belong to class III if the corresponding product $E(s)$ has only positive zeros and if $\sum_{1}^{\infty} a_{k}^{-1}<\infty$. The following result may be proved exactly as Theorem 4 was proved. The analogue of Theorem 2 which is needed here reduces to a special case of a well known result, see [5; pp. 322-327].

THEOREM 6. If

1. $G(t) \in$ class III,

2. $f(x)=\int_{-\infty}^{\infty} G(x-t) d \alpha(t)$ is defined for $x>T+b+\sum_{1}^{\infty} a_{n}^{-1}$,

3. $f(x)=0\left(x>\rho+b+\sum_{1}^{\infty} a_{n}^{-1}, \rho \geqq T\right)$, then $\alpha(t)$ is constant for $(\rho<t<\infty)$.

\section{BIBLIOGRAPHY}

1. I. I. Hirschman, Jr., A new representation and inversion theory for the Laplace integral, Duke Math. J. vol. 15 (1948) pp. 473-494.

2. I. I. Hirschman, Jr., and D. V. Widder, An inversion and representation theory for convolu- 
tion transforms with totally positive kernels, Proc. Nat. Acad. Sci. U.S.A. vol. 34 (1948) pp. 152-156.

3. - The inversion of a general class of convolution transforms, Trans. Amer. Math. Soc. vol. 66 (1949) pp. 135-201.

4. I. J. Schoenberg, On totally positive functions, Laplace integrals, and entire functions of the Laguerre-Pólya-Schur type, Proc. Nat. Acad. Sci. U.S.A. vol. 33 (1947) pp. 11-17.

5. E. C. Titchmarsh, Introduction to the theory of Fourier integrals, Oxford University Press, 1937.

6. D. V. Widder, The Laplace transform, Princeton University Press, 1941.

HARVARD UNIVERSITY,

Cambridge, Mass. 